\title{
Eco-friendly synthesis of fluorine-containing pyrazoline derivatives over potassium carbonate
}

\author{
Vimesh M. Patel and Kishor R. Desai* \\ Department of Chemistry, South Gujarat University, Surat-395007, India \\ E-mail:vmp_tithal@yahoo.co.in \\ (received 17 Mar 04; accepted 27 May 04; published on the web 06 Jun 04)
}

\begin{abstract}
Ten new fluorine-containing 1-thiocarbamoyl-3,5-diphenyl-2-pyrazolines $\mathbf{4 a - j}$ have been synthesized in $80-85 \%$ yield by a microwave-promoted solvent-free condensation of 2,4dichloro-5-fluoro chalcones $\mathbf{3 a - j}$ with thiosemicarbazide over potassium carbonate. The work-up is simple and involves treatment with ice-cold water. A considerable increase in the reaction rate has been observed, with better yields.
\end{abstract}

Keywords: Pyrazolines, solid-phase synthesis, condensation, chalcones, microwave effect

\section{Introduction}

Pyrazolines have been reported to show a broad spectrum of biological activities including antibacterial, ${ }^{1}$ antifungal, ${ }^{2}$ anti-inflammatory, ${ }^{3}$ and antidepressant activities. ${ }^{4}$ The pyrazoline function is quite stable, and has inspired chemists to utilize this stable fragment in bioactive moieties to synthesize new compounds possessing biological activities, and the presence of fluorine in the molecules at strategic positions alters the activity. This prompted us to synthesize various substituted pyrazoline derivatives using the microwave-assisted method. The most straightforward protocol for the synthesis of 1-thiocarbamoyl-3,5-diphenyl-2-pyrazolines $\mathbf{4 a - j}$ involves the one-pot condensation of chalcones $\mathbf{3 a - j}$ with thiosemicarbazide in ethanol under strongly basic conditions. ${ }^{5}$ However, the combination of solvents, strong base, and long reaction time period makes this method environmentally hazardous. Thus, a simple, general and efficient procedure for the synthesis of this important heterocyclic system is required. Furthermore, there is no report on the synthesis of 1-thiocarbamoyl-3,5-diphenyl-2-pyrazolines from 2,4-dichloro-5fluorochalcones using potassium carbonate under microwave irradiation (MWI). Therefore the development of new methods that lead to convenient procedures and better yields are of interest.

In the last few years Microwave-induced Organic Reaction Enhancement (MORE) chemistry has gained popularity as a non-conventional technique for rapid organic synthesis ${ }^{6}$ and many researchers have described accelerated organic reactions, and a large number of papers has 
appeared proving the synthetic utility of MORE chemistry in routine organic synthesis. ${ }^{7,8}$ It can be termed as 'e-chemistry' because it is easy, effective, economical and eco-friendly and is believed to be a step towards green chemistry.

Under the framework of, "Green Chemistry" we have developed an environmentally benign solvent-free approach for the synthesis of pyrazolines. This permitted the elimination of solvents and strong mineral bases in solution. ${ }^{9}$ Further attractions of this method are that it permits reactions in open vessels (thus avoiding the risk of high pressure developing) and synthesis on preparative scales. ${ }^{10}$ In view of the above, and in continuation to our earlier work on the application of $\mathrm{MORE}^{11}$ chemistry to organic synthesis and the biological importance of pyrazolines, we now report a simple microwave synthesis of pyrazolines $\mathbf{4 a}-\mathbf{j}$ from chalcones 3a-j and thiosemicarbazide over potassium carbonate $\left(\mathrm{K}_{2} \mathrm{CO}_{3}\right)$.

The desired chalcones ${ }^{12} \mathbf{3 a - j}$ were synthesized by reacting 2,4-dichloro-5fluoroacetophenone $\mathbf{1}$ with aromatic aldehydes $\mathbf{2 a}-\mathbf{j}$ in the presence of alkali. In a typical case, equimolar quantities of chalcones $\mathbf{3 a}-\mathbf{j}$ and thiosemicarbazide were adsorbed over $\mathrm{K}_{2} \mathrm{CO}_{3}$ (Method A) and subjected to MWI, which led to the formation of pyrazolines 4a-j. (Scheme 1). For a comparative study the reactants were adsorbed on basic alumina (Method B) and irradiated in a microwave oven (Table 1). The elution of product from a $1 \mathrm{~g}$ reaction batch, with basic alumina requires about $25-30 \mathrm{ml}$ of acetone, whereas with $\mathrm{K}_{2} \mathrm{CO}_{3}$ only water is required. This eliminates the use of organic solvent from the workup stage. The reaction time has been brought down from hours to minutes with improved yields using MWI. However, the yields of both methods (A and B) are equally satisfactory.

Table 1. Microwave-assisted $\mathrm{K}_{2} \mathrm{CO}_{3}$ / basic alumina-mediated synthesis of $\mathbf{4 a - j}$

\begin{tabular}{|c|c|c|c|c|c|}
\hline \multirow[b]{2}{*}{ Product } & \multirow[b]{2}{*}{$\mathrm{R}$} & \multicolumn{2}{|c|}{ Method $\mathrm{A} / \mathrm{K}_{2} \mathrm{CO}_{3}$} & \multicolumn{2}{|c|}{ Method B / basic alumina } \\
\hline & & $\begin{array}{l}\text { Time } \\
(\min )\end{array}$ & $\begin{array}{c}\text { Yield }^{\mathrm{a}} \\
(\%)\end{array}$ & $\begin{array}{l}\text { Time } \\
(\mathrm{min})\end{array}$ & $\begin{array}{c}\text { Yield }^{\mathrm{a}} \\
(\%)\end{array}$ \\
\hline $4 a$ & $\mathrm{H}$ & 9.0 & 82 & 5.5 & 82 \\
\hline $4 b$ & $2-\mathrm{NO}_{2}$ & 8.0 & 82 & 6 & 82 \\
\hline 4c & $3-\mathrm{NO}_{2}$ & 7.5 & 82 & 6 & 80 \\
\hline $4 d$ & $2-\mathrm{Cl}$ & 7.0 & 85 & 5 & 85 \\
\hline $4 e$ & $4-\mathrm{Cl}$ & 8.5 & 81 & 5.5 & 80 \\
\hline $4 f$ & $4-\mathrm{N}\left(\mathrm{CH}_{3}\right)_{2}$ & 7.0 & 80 & 5 & 81 \\
\hline $4 g$ & $4-\mathrm{OCH}_{3}, 3-\mathrm{OH}$ & 7.0 & 82 & 6 & 80 \\
\hline $4 h$ & $3,4,5-\left(\mathrm{OCH}_{3}\right)_{3}$ & 9.0 & 82 & 5 & 85 \\
\hline $4 I$ & $3-\mathrm{OC}_{6} \mathrm{H}_{5}$ & 9.0 & 60 & 5.5 & 62 \\
\hline $4 j$ & $4-\mathrm{OCH}_{3}$ & 8.0 & 80 & 6 & 80 \\
\hline
\end{tabular}

${ }^{\mathrm{a}}$ Yield of isolated products. 


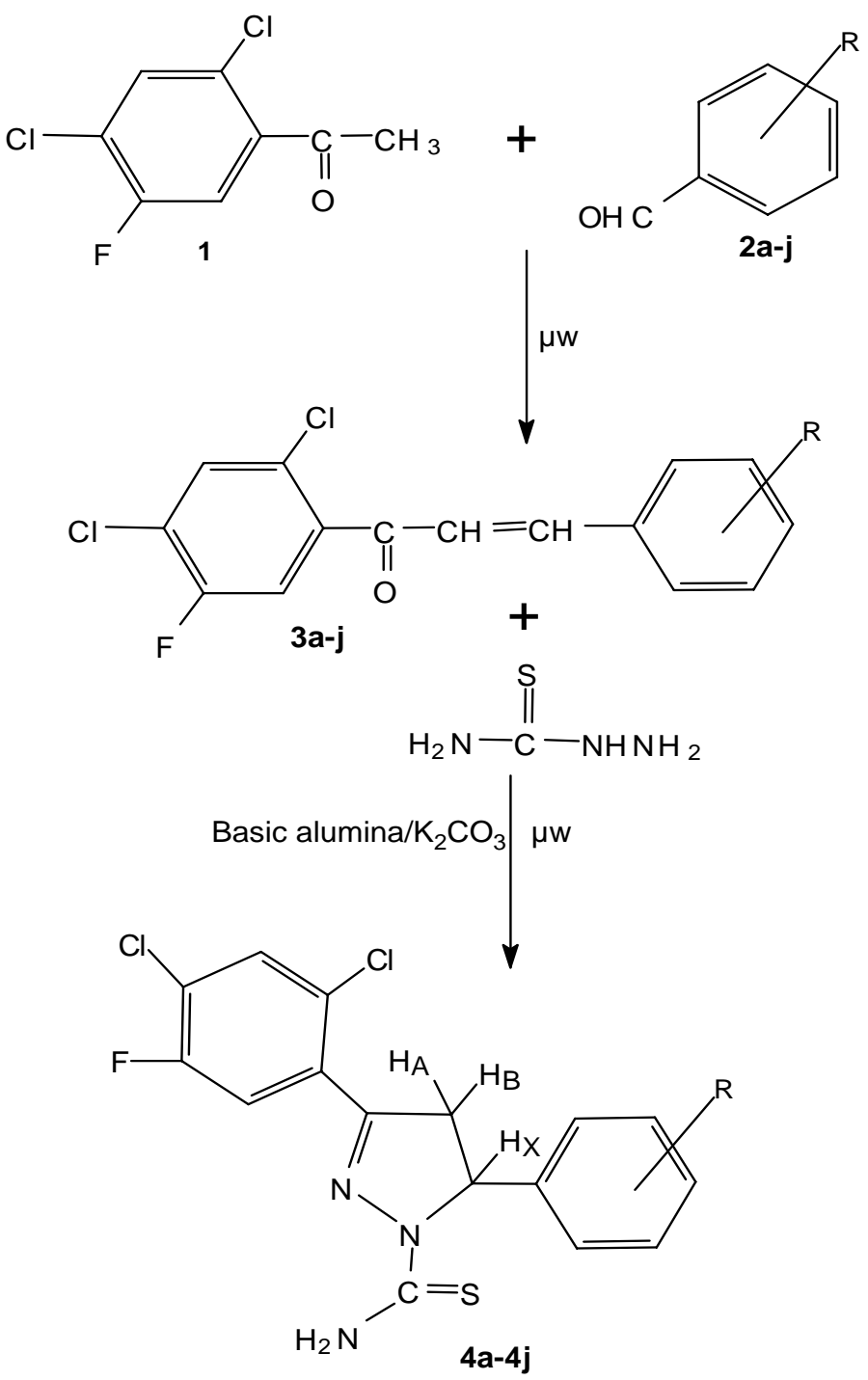

Scheme 1. Synthesis of pyrazolines under MWI.

Finally, the synthesis of $4 a$ using $\mathrm{K}_{2} \mathrm{CO}_{3}$ under neat conditions was attempted using a thermostatted oil-bath under identical conditions as those employed as for the microwaveassisted method $\left(9 \mathrm{~min}\right.$ and $\left.120^{\circ} \mathrm{C}\right)$. Lower yields were obtained under thermal conditions (27\%) as compared to microwave irradiation $(82 \%)$, demonstrating that the effect of microwave irradiation is not purely thermal. Microwave irradiation facilitates the polarization of the molecules under irradiation, causing rapid reaction to occur. This is consistent with the reaction mechanism, which involves a polar transition state. ${ }^{13}$ Nucleophilic attack of an amine on a polarized carbonyl function (rate-determining step) is followed by an intramolecular cyclization.

In general, the reactions are fast, clean, and high yielding except for that leading to product 4i, Table 1, where 1-thiocarbamoyl-3-(2,4-dichloro-5-fluorophenyl)-5-(3-phenoxyphenyl)-2pyrazoline was obtained in $60 \%$ yield. (Scheme 2). When the corresponding 2,4-dichloro-5- 
fluorochalcones and thiosemicarbazide were subjected to microwave irradiation over $\mathrm{K}_{2} \mathrm{CO}_{3}$ $20 \%$ of the starting material was recovered. The same reaction with conventional methods gave $40 \%$ of the product, and $45 \%$ of the starting material was recovered.

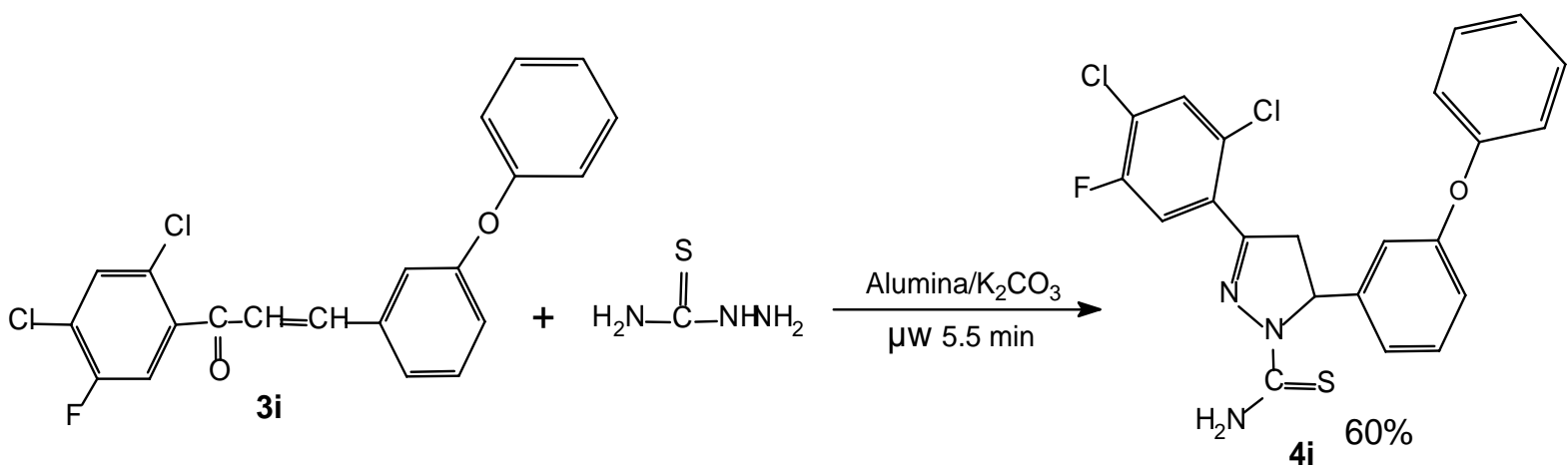

Scheme 2. Synthesis of product $4 \mathbf{i}$.

In conclusion, we have developed an easy, convenient and efficient synthetic methodology for pyrazoline derivatives using microwave assisted solid phase technique. The technique used herein can also be further elaborated for the synthesis of other diverse heterocyclic compounds. This rapid and easy technique coupled with solvent-free conditions may contribute to the dream of green technology.

\section{Experimental Section}

General Procedures. Melting points were determined in open capillary tubes and are uncorrected. The purities of the compounds were checked on silica-gel-coated Al plates (Merck). IR spectra were recorded in $\mathrm{KBr}$ on a Perkin Elmer Spectrum BX series FT-IR spectrophotometer. ${ }^{1} \mathrm{H}-\mathrm{NMR}$ spectra were recorded on Bruker DRX $300 \mathrm{MHz}$ NMR spectrometer using TMS as internal standard and mass spectra on a Jeol D-300 spectrometer. Elemental analyses were carried out on a Carlo Erba 1108 analyzer. Microwave irradiations were carried out in a Q-PRO M microwave synthesis system with power output of 800 watts.

Chalcones (3a-j). Chalcone derivatives were synthesized by condensing 2,4-dichloro-5fluoroacetophenone 1 with various aldehydes $2 \mathbf{a}-\mathbf{j}$ according to the method reported in the literature. $^{12}$

\section{Microwave / $\mathrm{K}_{2} \mathrm{CO}_{3}$ mediated synthesis of pyrazolines $4 \mathrm{a}-\mathrm{j}$ (Method $\mathrm{A}$ )}

A mixture of the chalcone $\mathbf{3 a}-\mathbf{j}(2.2 \mathrm{mmol})$ and thiosemicarbazide $(2 \mathrm{mmol})$ was dissolved in acetone $(5 \mathrm{~mL})$ and ethanol $(5 \mathrm{~mL})$, then $\mathrm{K}_{2} \mathrm{CO}_{3}(4.0 \mathrm{~g})$ was added and stirred vigorously. After 5 
min, the solvent was removed under vacuum and the dry powder was irradiated in a microwave oven for the appropriate time (Table) at $650 \mathrm{~W}$. After completion of reaction as followed by t.l.c. examination, chilled water was added to the reaction mixture. The solid product was obtained, which was filtered, dried and crystallized from a suitable solvent (acetone-ethanol).

\section{Microwave/basic-alumina-mediated synthesis of pyrazolines 4a-j (Method B)}

A mixture of the chalcone $3 \mathbf{a}-\mathbf{j}(2.2 \mathrm{mmol})$ and thiosemicarbazide $(2 \mathrm{mmol})$ was dissolved in acetone $(5 \mathrm{~mL})$ and ethanol $(5 \mathrm{~mL})$ respectively. Basic alumina $(4 \mathrm{~g})$ was added and stirred vigorously. After $5 \mathrm{~min}$, the solvent was removed under vacuum and the dry powder was irradiated in a microwave oven for the appropriate time (Table) at $650 \mathrm{~W}$. After completion of the reaction (followed by t.l.c.) the product was eluted with acetone $(30 \mathrm{~mL})$. Removal of the solvent under reduced pressure yielded the product which was recrystallized from (acetoneethanol). The structures of the products were confirmed by elemental analysis, IR, ${ }^{1} \mathrm{H}-\mathrm{NMR}$, mass spectral data, and comparison with authentic samples prepared according to literature methods. $^{5,12}$

1-Thiocarbamoyl-3-(2,4-dichloro-5-fluorophenyl)-5-phenyl-2-pyrazoline (4a). m.p. $165^{\circ} \mathrm{C}$; IR (KBr): 3340, 1560, 1340, $1080 \mathrm{~cm}^{-1} ;{ }^{1} \mathrm{H}-\mathrm{NMR}$ (300 MHz, $\left.\mathrm{CDCl}_{3}-\mathrm{DMSO}-d_{6}\right): \delta 3.28$ (dd, $1 \mathrm{H}$, $\left.\mathrm{H}_{\mathrm{A}}\right), 3.90\left(\mathrm{dd}, 1 \mathrm{H}, \mathrm{H}_{\mathrm{B}}\right), 6.18\left(\mathrm{dd}, 1 \mathrm{H}, \mathrm{H}_{\mathrm{X}}\right), 6.71\left(\right.$ br. s, $\left.2 \mathrm{H}, \mathrm{NH}_{2}\right), 6.91-7.80$ (m, 7H, $\mathrm{H}_{\text {arom}}$ ); MS (EI): $\mathrm{m} / \mathrm{z}(\%) 356$ (31.72) $\left[\mathrm{M}^{+}\right], 357$ (100) $\left[\mathrm{M}+\mathrm{H}^{+}\right]$; Anal. Calcd. for $\mathrm{C}_{16} \mathrm{H}_{12} \mathrm{~N}_{3} \mathrm{SCl}_{2} \mathrm{~F}: \mathrm{C}, 52.18$; H, 3.28; N, 11.41. Found: C, 52.11; H, 3.35; N, 11.45.

1-Thiocarbamoyl-3-(2,4-dichloro-5-fluorophenyl)-5-(2-nitrophenyl)-2-pyrazoline (4b). m.p. $153^{\circ} \mathrm{C}$; IR (KBr): $3350,1545,1360,1335,1090 \mathrm{~cm}^{-1}$; ${ }^{1} \mathrm{H}-\mathrm{NMR}\left(300 \mathrm{MHz}, \mathrm{CDCl}_{3}-\mathrm{DMSO}-d_{6}\right): \delta$ $3.25\left(\mathrm{dd}, 1 \mathrm{H}, \mathrm{H}_{\mathrm{A}}\right), 3.91\left(\mathrm{dd}, 1 \mathrm{H}, \mathrm{H}_{\mathrm{B}}\right), 6.15\left(\mathrm{dd}, 1 \mathrm{H}, \mathrm{H}_{\mathrm{X}}\right), 6.75$ (br. s, 2H, $\left.\mathrm{NH}_{2}\right), 6.95-7.65(\mathrm{~m}, 6 \mathrm{H}$, $\left.\mathrm{H}_{\text {arom}}\right)$; $\mathrm{MS}$ (EI): $\mathrm{m} / \mathrm{z} \quad(\%) 356$ (31.72) $\quad\left[\mathrm{M}^{+}\right], 357 \quad$ (100) $\left[\mathrm{M}+\mathrm{H}^{+}\right]$; Anal. Calcd. for $\mathrm{C}_{16} \mathrm{H}_{11} \mathrm{~N}_{4} \mathrm{SO}_{2} \mathrm{Cl}_{2} \mathrm{~F}$ : C, 46.50; H, 2.68; N, 13.56. Found: C, 46.45; H, 2.61; N, 13.59.

1-Thiocarbamoyl-3-(2,4-dichloro-5-fluorophenyl)-5-(3-nitrophenyl)-2-pyrazoline (4c). m.p. $160^{\circ} \mathrm{C}$; IR (KBr): $3295,1555,1370,1340,1085 \mathrm{~cm}^{-1}$; ${ }^{1} \mathrm{H}-\mathrm{NMR}\left(300 \mathrm{MHz}, \mathrm{CDCl}_{3}-\mathrm{DMSO}-d_{6}\right): \delta$ $3.29\left(\mathrm{dd}, 1 \mathrm{H}, \mathrm{H}_{\mathrm{A}}\right), 3.95\left(\mathrm{dd}, 1 \mathrm{H}, \mathrm{H}_{\mathrm{B}}\right), 6.12\left(\mathrm{dd}, 1 \mathrm{H}, \mathrm{H}_{\mathrm{X}}\right), 6.65\left(\right.$ br. s, $\left.2 \mathrm{H}, \mathrm{NH}_{2}\right), 6.90-7.50(\mathrm{~m}, 6 \mathrm{H}$, $\mathrm{H}_{\text {arom}}$ ); Anal. Calcd. for $\mathrm{C}_{16} \mathrm{H}_{11} \mathrm{~N}_{4} \mathrm{SO}_{2} \mathrm{Cl}_{2} \mathrm{~F}$ : C, 46.50; H, 2.68; N, 13.56. Found: C, 46.41; H, $2.62 ; \mathrm{N}, 13.58$.

1-Thiocarbamoyl-3-(2,4-dichloro-5-fluorophenyl)-5-(2-chlorophenyl)-2-pyrazoline

(4d). m.p. $145^{\circ} \mathrm{C}$; IR (KBr): 3300, 1575, 1380, 1345, $1100 \mathrm{~cm}^{-1}$; ${ }^{1} \mathrm{H}-\mathrm{NMR}$ (300 MHz, $\mathrm{CDCl}_{3}-\mathrm{DMSO}-$ $\left.d_{6}\right): \delta 3.35\left(\mathrm{dd}, 1 \mathrm{H}, \mathrm{H}_{\mathrm{A}}\right), 3.91\left(\mathrm{dd}, 1 \mathrm{H}, \mathrm{H}_{\mathrm{B}}\right), 6.16\left(\mathrm{dd}, 1 \mathrm{H}, \mathrm{H}_{\mathrm{X}}\right), 6.75$ (br. s, 2H, NH $), 7.10-7.32$ (m, 6H, $\mathrm{H}_{\text {arom }}$ ); MS (EI): m/z (\%) = $390.5(100)\left[\mathrm{M}^{+}\right]$; Anal. Calcd. for $\mathrm{C}_{16} \mathrm{H}_{11} \mathrm{~N}_{3} \mathrm{SCl}{ }_{3} \mathrm{~F}: \mathrm{C}, 47.72$; H, 2.75; N, 10.43. Found: C, 47.68; H, 2.78; N, 10.47.

1-Thiocarbamoyl-3-(2,4-dichloro-5-fluorophenyl)-5-(4-chlorophenyl)-2-pyrazoline (4e). m.p. $160^{\circ} \mathrm{C}$; IR (KBr): 3280, 1570, 1390, 1310, $1110 \mathrm{~cm}^{-1}$; ${ }^{1} \mathrm{H}-\mathrm{NMR}\left(300 \mathrm{MHz}, \mathrm{CDCl}_{3}-\mathrm{DMSO}-\right.$ $\left.d_{6}\right): \delta 3.41\left(\mathrm{dd}, 1 \mathrm{H}, \mathrm{H}_{\mathrm{A}}\right), 4.10\left(\mathrm{dd}, 1 \mathrm{H}, \mathrm{H}_{\mathrm{B}}\right), 6.21\left(\mathrm{dd}, 1 \mathrm{H}, \mathrm{H}_{\mathrm{X}}\right), 6.80$ (br. s, $\left.2 \mathrm{H}, \mathrm{NH}_{2}\right), 7.23-7.60$ $\left(\mathrm{m}, 6 \mathrm{H}, \mathrm{H}_{\text {arom }}\right) ; \mathrm{MS}(\mathrm{EI}): \mathrm{m} / \mathrm{z}(\%)=356(31.72)\left[\mathrm{M}^{+}\right], 357(100)\left[\mathrm{M}+\mathrm{H}^{+}\right]$; Anal. Calcd. for $\mathrm{C}_{16} \mathrm{H}_{11} \mathrm{~N}_{3} \mathrm{SCl}_{3} \mathrm{~F}$ : C, 47.72; H, 2.75; N, 10.43. Found: C, 47.67; H, 2.77; N, 10.48 . 
1-Thiocarbamoyl-3-(2,4-dichloro-5-fluorophenyl)-5-(4- $\boldsymbol{N}, \boldsymbol{N}$-dimethylaminophenyl)-2-

pyrazoline (4f). m.p. $150^{\circ} \mathrm{C}$; IR (KBr): $3365,2875,1600,1395,1290,1120 \mathrm{~cm}^{-1} ;{ }^{1} \mathrm{H}-\mathrm{NMR}(300$ $\left.\mathrm{MHz}, \mathrm{CDCl}_{3}-\mathrm{DMSO}-\mathrm{d}_{6}\right): \delta 2.46\left(\mathrm{~s}, 6 \mathrm{H}, \mathrm{N}\left(\mathrm{CH}_{3}\right)_{2}\right), 3.45\left(\mathrm{dd}, 1 \mathrm{H}, \mathrm{H}_{\mathrm{A}}\right), 4.25\left(\mathrm{dd}, 1 \mathrm{H}, \mathrm{H}_{\mathrm{B}}\right), 6.12(\mathrm{dd}$, $1 \mathrm{H}, \mathrm{H}_{\mathrm{X}}$ ), 6.82 (br. s, $2 \mathrm{H}, \mathrm{NH}_{2}$ ), 7.08-7.51 (m, 6H, $\mathrm{H}_{\text {arom}}$ ); Anal. Calcd. for $\mathrm{C}_{18} \mathrm{H}_{17} \mathrm{~N}_{4} \mathrm{SCl}_{2} \mathrm{~F}$ : C, 52.56; H, 4.17; N, 13.62. Found: C, 52.51; H, 4.11; N, 13.65.

1-Thiocarbamoyl-3-(2,4-dichloro-5-fluorophenyl)-5-(2-nitrophenyl)-2-pyrazoline (4g). m.p. $125^{\circ} \mathrm{C}$; IR (KBr): 3590, 3340, 2870, 1580, 1360, 1280, $1110 \mathrm{~cm}^{-1} ;{ }^{1} \mathrm{H}-\mathrm{NMR}\left(300 \mathrm{MHz}, \mathrm{CDCl}_{3}-\right.$ DMSO-d $\left.)_{6}\right): \delta 3.25\left(\mathrm{dd}, 1 \mathrm{H}, \mathrm{H}_{\mathrm{A}}\right), 3.61(\mathrm{dd}, 1 \mathrm{H}, \mathrm{OH}), 3.82\left(\mathrm{~s}, 3 \mathrm{H}, \mathrm{OCH}_{3}\right), 3.95\left(\mathrm{dd}, 1 \mathrm{H}, \mathrm{H}_{\mathrm{B}}\right) 6.17$ $\left(\mathrm{dd}, 1 \mathrm{H}, \mathrm{H}_{\mathrm{X}}\right), 6.75$ (br. $\left.\mathrm{s}, 2 \mathrm{H}, \mathrm{NH}_{2}\right), 6.95-7.35\left(\mathrm{~m}, 5 \mathrm{H}, \mathrm{H}_{\text {arom }}\right)$; Anal. Calcd. for $\mathrm{C}_{17} \mathrm{H}_{14} \mathrm{~N}_{3} \mathrm{SO}_{2} \mathrm{Cl}_{2} \mathrm{~F}$ : C, 49.29; H, 3.41; N, 10.14. Found: C, 49.32; H, 3.48; N, 10.09 .

1-Thiocarbamoyl-3-(2,4-dichloro-5-fluorophenyl)-5-(3,4,5-trimethoxyphenyl)-2-pyrazoline (4h). m.p. $168^{\circ} \mathrm{C}$; IR (KBr): 3350, 2977, 1562, 1345, $1082 \mathrm{~cm}^{-1} ;{ }^{1} \mathrm{H}-\mathrm{NMR}\left(300 \mathrm{MHz}, \mathrm{CDCl}_{3}-\right.$ DMSO-d $\left.)_{6}\right): \delta 3.18\left(\mathrm{dd}, 1 \mathrm{H}, \mathrm{H}_{\mathrm{A}}\right), 3.81\left(\mathrm{~s}, 9 \mathrm{H}, 3 \mathrm{xOCH}_{3}\right), 3.98\left(\mathrm{dd}, 1 \mathrm{H}, \mathrm{H}_{\mathrm{B}}\right), 6.19\left(\mathrm{dd}, 1 \mathrm{H}, \mathrm{H}_{\mathrm{X}}\right)$,

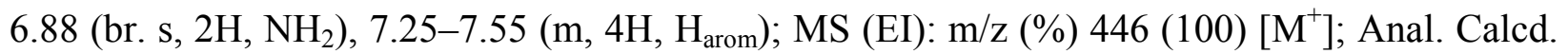
for $\mathrm{C}_{19} \mathrm{H}_{18} \mathrm{~N}_{3} \mathrm{SO}_{3} \mathrm{Cl}_{2} \mathrm{~F}$ : C, 49.79; H, 3.96; N, 9.17. Found: C, 49.71; H, 3.99; N, 9.11.

1-Thiocarbamoyl-3-(2,4-dichloro-5-fluorophenyl)-5-(3-phenoxyphenyl)-2-pyrazoline (4i). m.p. $165^{\circ} \mathrm{C}$; IR (KBr): 3340, 1547, 1365, 1335, $1095 \mathrm{~cm}^{-1}$; ${ }^{1} \mathrm{H}-\mathrm{NMR}\left(300 \mathrm{MHz}, \mathrm{CDCl}_{3}-\mathrm{DMSO}-d_{6}\right): \delta$ $3.28\left(\mathrm{dd}, 1 \mathrm{H}, \mathrm{H}_{\mathrm{A}}\right), 3.99\left(\mathrm{dd}, 1 \mathrm{H}, \mathrm{H}_{\mathrm{B}}\right), 6.18$ (dd, 1H, $\left.\mathrm{H}_{\mathrm{X}}\right), 6.70$ (br. s, 2H, $\left.\mathrm{NH}_{2}\right), 6.91-7.48(\mathrm{~m}, 7 \mathrm{H}$, $\left.\mathrm{H}_{\text {arom}}\right) ; \mathrm{MS}$ (EI): $\mathrm{m} / \mathrm{z} \quad(\%) 448$ (31.12) $\quad\left[\mathrm{M}^{+}\right], 449$ (100) $\left[\mathrm{M}+\mathrm{H}^{+}\right]$; Anal. Calcd. for $\mathrm{C}_{22} \mathrm{H}_{16} \mathrm{~N}_{3} \mathrm{SOCl}_{2} \mathrm{~F}$ : C, 57.40; H, 3.50; N, 9.13. Found: C, 57.41; H, 3.55; N, 9.17.

1-Thiocarbamoyl-3-(2,4-dichloro-5-fluorophenyl)-5-(2-nitrophenyl)-2-pyrazoline (4j). m.p. $165^{\circ} \mathrm{C}$; IR (KBr): $3375,2890,1590,1370,1115 \mathrm{~cm}^{-1}$; ${ }^{1} \mathrm{H}-\mathrm{NMR}\left(300 \mathrm{MHz}, \mathrm{CDCl}_{3}-\mathrm{DMSO}-d_{6}\right): \delta$ $3.26\left(\mathrm{dd}, 1 \mathrm{H}, \mathrm{H}_{\mathrm{A}}\right), 3.85\left(\mathrm{~s}, 3 \mathrm{H}, \mathrm{OCH}_{3}\right), 3.99\left(\mathrm{dd}, 1 \mathrm{H}, \mathrm{H}_{\mathrm{B}}\right), 6.19$ (dd, $\left.1 \mathrm{H}, \mathrm{H}_{\mathrm{X}}\right), 6.75$ (br. s, $2 \mathrm{H}$, $\mathrm{NH}_{2}$ ), 6.95-7.34 (m, 7H, $\mathrm{H}_{\text {arom}}$ ); Anal. Calcd. for $\mathrm{C}_{17} \mathrm{H}_{14} \mathrm{~N}_{3} \mathrm{SOCl}_{2} \mathrm{~F}$ : C, 51.26; H, 3.54; N, 10.55 . Found: C, 51.29; H, 3.51; N, 10.59 .

\section{Acknowledgements}

The authors are thankful to the South Gujarat University, Surat for providing research facilities and CDRI, Lucknow for providing ${ }^{1} \mathrm{H}-\mathrm{NMR}$ and mass spectra respectively.

\section{References}

1. Nauduri, D.; Reddy, G. B. Chem. Pharm. Bull. Tokyo 1998, 46, 1254.

2. Korgaokar, S. S.; Patil, P. H.; Shah, M. T.; Parekh, H. H. Indian J. Pharm. Sci. 1996, 58, 222.

3. Udupi, R. H.; Kushnoor, A. R.; Bhat, A. R. Indian J. Heterocycl. Chem. 1998, 8, 63.

4. Bilgin, A. A.; Palaska, E.; Sunal, R. Arzneimforsch Drug Res. 1993, 43, 1041. 
5. Malhotra, V.; Pathak, S.; Nath, R.; Mukerjee, D.; Shanker, K. Indian J. Chem. 2002, 41B, 1310.

6. Varma, S. Green Chemistry 1999, 1, 43.

7. Borah, R.; Kalita, D. J.; Sarma J. C. Indian J. Chem. 2002, 41B, 1032.

8. Kidwai, M.; Dave, B.; Venkataramanan, R. Indian J. Chem. 2002, 41B, 2414.

9. Loupy, A. In Topics in Current Chemistry: Modern Solvents in Organic Synthesis, Knochel, P., Ed.; Springer Verlag: New York, 1999; Vol. 206, p 153.

10. Cleophax, J.; Liagre, M.; Loupy, A.; Petit, A. Org. Process Res. Dev. 2000, 6, 498.

11. Patel, V. M.; Desai, K. R. Indian J. Chem. 2004, 43B, 191.

12. Gupta, R.; Gupta, A. K.; Paul, S.; Kachroo, P. L. Indian J. Chem. 1995, 34B, 61.

13. Loupy, A.; Perreux, L.; Liagre, M.; Burle, K.; Moneuse, M. Pure Appl. Chem. 2001, 73, 161. 